\title{
Biochemical Effect of Artemisin-based Combination Therapy (ACT) Antimalarial Drugs on Enzymatic Antioxidants in
}

\section{Pregnancy}

\section{Oyeyemi A01, Ayeni AM11, Oyeyemi RB², Aluko BT1* and Ogunsile $0 J^{3}$}

${ }^{1}$ Department of Biochemistry, Ekiti State University, Nigeria

${ }^{2}$ Human Resource Department, Procter \& Gamble Nigeria, Nigeria

${ }^{3}$ Biochemistry Department, University of Mysore, India

\section{Research Article \\ Volume 4 Issue 1}

Received Date: March 20, 2019

Published Date: March 29, 2019

DOI: $10.23880 /$ act-16000151

${ }^{*}$ Corresponding author: Aluko Bukola, Department of Biochemistry, Ekiti State University, Ekiti State, Nigeria, Tel: +2348038686409; Email: bukola.aluko3@gmail.com

\section{Abstract}

This study was undertaken to determine the effect of artemisin-based combination therapy (ACT) on Plasma enzymatic antioxidants in pregnant women with malaria. Seventy-five (75) consenting pregnant subjects (25malarial infected pregnant women receiving ACT, 25 malarial infected pregnant women yet to be treated and 25 pregnant women without malarial infection) were randomly selected. The activities of the plasma enzymatic antioxidants were determined. Glutathione peroxidase (GPx), superoxide dismutase (SOD), and Catalase (CAT) were the enzymatic antioxidants determined using spectrophotometry method. Results showed that Plasmodium falciparum malarial infection significantly reduced $(\mathrm{p}<0.05)$ plasma enzymatic antioxidants in pregnant patients without ACT treatments as compared with values obtained in pregnant patients on ACT. The pregnant patients without malaria infection served as the control. Thus, this study suggests that the treatment of Plasmodium falciparum malarial infection with ACT in pregnancy significantly $(\mathrm{p}<0.05)$ improves the activities of all the plasma antioxidants determined.

Keywords: Artemisin-Based Combination Therapy (ACT); Malaria, Catalase (CAT); Superoxide Dismutase (SOD); Glutathione Peroxidase (Gpx)

\section{Introduction}

Malaria is an old disease whose name is derived from the Italian (mal-aria) or "bad air" and it was also known as Roman fever, ague, marsh fever or periodic fever. There were numerous, sometimes bizarre theories on how malaria was transmitted until 1898 when Dr. Ronald Ross discovered that the female Anopheles mosquito was actually responsible for transmitting malaria parasite. This discovery revolutionized malaria control, which had hitherto often been haphazard or based purely on treating the patient by killing the malaria parasites [1]. 
Malaria is a life threatening protozoan disease caused by malaria parasites belonging to the genus Plasmodium. Malaria parasites infecting humans belong to four species: Plasmodium (P.) falciparum, P. vivax, P. malariae and P.ovale. While these four species do not ordinarily infect animals; there is evidence that Chimpanzees may act as a reservoir host for Plasmodium malariae in Africa, providing a possible source of human infection [2].

Malaria is a life threatening parasitic disease transmitted by female Anopheles mosquitoes. More than $40 \%$ of the world population lives in malarious areas. It is estimated that the number of cases of malaria rose from 233 million in 2000 to 244 million in 2005 but decreased to 225 million in 2009. The number of deaths due to malaria is estimated to have decreased from 985,000 in 2000 to 781,000 in 2009. Malaria is the most highly prevalent tropical disease with high morbidity and mortality, and with high economic and social impact. Over $90 \%$ of all deaths caused by malaria occur in sub Saharan Africa and about $85 \%$ of deaths globally were in children under 5 years of age [3]. In addition, pregnant women are at immense risk of malaria due to natural immune depression in pregnancy [4]. About $25 \%$ of all estimated malaria cases in the World Health Organization African Region occur in Nigeria [3].

Malaria during pregnancy continues to be a major health problem in endemic countries with clinical consequences including death of both mother and child [5]. Malaria remains the single most important infection causing morbidity and mortality in the world and is second only to Mycobacterium tuberculosis as the single most important infection agent [6]. It is one of the biggest impediments to progress in Africa and is the biggest killer in Africa, with $90 \%$ of the global malaria deaths occurring in this continent. It is responsible for one in four deaths below the age of 5 years and could most times lead to miscarriage at the early stage of pregnancy [7]. In the endemic countries of Africa, children under the age of five and pregnant women bear the brunt of the burden of malaria disease, this is because they have lower immunity to the disease compared to other people in the same environment [8].

Antioxidant enzyme plays an important role against oxidative stress by enzymatic neutralization of hydrogen peroxide to oxygen and water [9]. These substances include some metallo-enzymes like catalase, superoxide dismutase (SOD) and glutathione peroxidase. Many antioxidant micronutrients have been found to improve immune functions in many animals including human beings, and they act by donating electrons or binding directly to the free radicals, or interrupting the chain reactions involved in cell damage [10].

Catalase is an important enzyme in protecting the cell from oxidative damage by reactive oxygen species (ROS) likewise, it is one of the highest turnover numbers of all enzymes; one catalase molecule can convert approximately 5 million molecules of hydrogen peroxide to water and oxygen each second [11]. Superoxide dismutase (SOD) is an antioxidant enzyme that catalyses the dismutation of superoxide anions into hydrogen peroxide and molecular oxygen. SOD plays important protective roles against cellular and histological damages that are produced by ROS. Superoxide reacts rapidly with nitric oxide (NO), reducing NO bioactivity and producing the oxidative peroxynitrite radical [10]. Gluthathione peroxidase (Gpx) is an antioxidant enzyme that catalyses the reduction of reactive oxygen species (ROS) to water.

Despite the enormous interventions, malaria is still a concern in most developing countries in Africa. Malaria is the most significant parasitic disease of humans, and claims the lives of more children worldwide than any other infectious disease and it is the third most infectious diseases plaguing the sub-Saharan regions with tuberculosis and diarrhoeal infections and has become a major public health issue and concern [12]. The emergence of resistant strains of Plasmodium falciparum to chloroquine and some antimalarial drugs led to the World Health Organization recommendation on the use of artemisinin combination therapy (ACT) as a first line treatment for uncomplicated malaria [13].

Artemisinin which is isolated from the plant Artemisia annua is a sesquiterpene lactone with an unusual peroxide bridge believed to be responsible for the therapeutic actions of the plant. Artemisinins not merely achieve prompt clearance of the asexual parasites that are responsible for symptomatic blood-stage infection; they also diminish the numbers of sexual-stage parasites (gametocytes) responsible for spread. Combined with a second, longer acting partner drug, the resultant artemisinin-based combination therapy (ACT) is expected to allow fewer opportunities for the progress of drug resistance as the artemisinin component kills the majority of parasites at the start of the treatment, while the more slowly metabolized partner drug clears the lingering parasites. The biggest challenge yet to the effective control of malaria is combating drug resistance. The 
parasite responsible for malaria ( $P$. falciparum) is becoming resistant even to ACT [14].

\section{Methodology}

\section{Subjects}

Subjects used for this study were made up of seventyfive (75) pregnant women, who attended antenatal and postnatal clinics of the Wesley Guide Hospital, Ilesha. Osun State, Nigeria. The subjects were divided into three (3) groups. Group one was made up of twenty-five (25) malaria infected pregnant women on ACT drugs. Group Two was made of twenty-five (25) malaria infected pregnant women not on ACT drugs. Group 3 was made up of pregnant women without malaria infection.

\section{Selection of Subjects}

The selection of Malaria subjects in this study was made by screening patients admitted from the antenatal and post natal wards of Wesley Guide Teaching Hospital, Ilesha. Osun State

Interview: An interview was conducted to obtain the socio demographic and reproductive health status of the subjects. These were obtained with the use of questionnaire, which provides all relevant information that ensured the choice of the right subjects and exclusion of subjects that were not fit for the study.

Anthropometric measurement: Height (metres) without shoes was measured with a wall-mounted ruler. Weight $(\mathrm{kg})$ in light clothing was measured with a bathroom scale (Zhongshan Camry Electronic Co. Ltd, Guangdong, China). Body mass index (BMI) was calculated using the formula; weight $(\mathrm{kg}) /$ height $\left(\mathrm{m}^{2}\right)$.

Ethical Approval: A letter of Identification from the Department of Biochemistry, Ekiti State University, AdoEkiti was used in pre-survey visits made to the hospital to obtain permission from the hospitals' authorities. Ethical clearance was also obtained. During the visits, the management, health workers in charge of antenatal services and laboratory scientists were informed on the nature and objectives of the study. They later organized and informed the pregnant women about the study. The informed consent of both management and the pregnant women were sought and obtained before the commencement of the study.
Collections of samples: All the subjects in each group while resting and supine had $10 \mathrm{ml}$ of their venous non fasting blood samples drawn by vein puncture from each woman into lithium heparinized anticoagulant bottles. The blood was immediately centrifuged at 4,000 revolutions per minute (rpm) for 15minutes, and separated to obtain the plasma. The samples were then analyzed to obtain the concentrations of all the desired parameters in this study.

Antioxidant assays: Superoxide dismutase activity was determined by the method of Marklund and Marklund [15]. Catalase activity was determined by the method described by Beers RF, et al. [16]. Gluthathione peroxidase activity was determined by the method described by Paglia DE, et al. [17].

Statistical Analysis: The results obtained was grouped and expressed as mean \pm Standard Error of Mean (SEM). The data collected was analyzed using one -way Analysis of variance (ANOVA) and Duncan multiple range test to compare the data obtained from the experiment to those of the control.

\section{Results}

\begin{tabular}{|c|c|c|}
\hline Parameters & Group 1 & Control \\
\hline (MMOL/L) & MEAN + SD & MEAN+ SD \\
\hline Gluthathione Peroxidase & $0.14+0.08 \mathrm{a}$ & $0.16+0.07 \mathrm{a}$ \\
\hline Superoxide Dismutase & $1.302+0.34 \mathrm{a}$ & $1.33+0.35 \mathrm{a}$ \\
\hline Catalase & $0.228+0.16 \mathrm{a}$ & $0.25+0.12 \mathrm{a}$ \\
\hline
\end{tabular}

Table 1: Activities of Enzymatic Antioxidants in Malaria infected Pregnant Women on ACT treatment and Pregnant Women without Malaria.

Results are expressed as mean and standard deviation. $\mathrm{P} \leq 0.05$ Test values carrying superscript (a) are not significantly different from the control. Group1: Malaria infected pregnant women on ACT treatment

Control: Pregnant women without malaria

\begin{tabular}{|c|c|c|}
\hline Parameters & Group 2 & Control \\
\hline (MMOL/L) & MEAN+ SD & MEAN+SD \\
\hline Gluthathione Peroxidase & $0.0018+0.04 \mathrm{~b}$ & $0.16+0.07 \mathrm{a}$ \\
\hline Superoxide Dismutase & $0.035+0.08 \mathrm{~b}$ & $1.33+0.35 \mathrm{a}$ \\
\hline Catalase & $0.025+0.06 \mathrm{~b}$ & $0.25+0.12 \mathrm{a}$ \\
\hline
\end{tabular}

Table 2: Activities of Enzymatic Antioxidants in Malaria infected Pregnant Women without ACT treatment And Pregnant Women without Malaria. 
Results are expressed as mean and standard deviation. $\mathrm{P} \leq 0.05$ Test values carrying superscript (b) are significantly different from the control.

Group 2: Malaria infected pregnant women without ACT treatment

Control: Pregnant women without malaria

\begin{tabular}{|c|c|c|c|}
\hline Parameters & Group 1 & Group 2 & Control \\
\hline (MMOL/L) & MEAN + SD & MEAN+ SD & MEAN+ SD \\
\hline Age (Years) & $30+3.32 \mathrm{a}$ & $30+3.32 \mathrm{a}$ & $30+3.32 \mathrm{a}$ \\
\hline Weight (Kg) & $65+3.32 \mathrm{a}$ & $55+3.32 \mathrm{a}$ & $70+3.32 \mathrm{a}$ \\
\hline Height (m2) & $1.8+0.16 \mathrm{a}$ & $1.3+0.16 \mathrm{a}$ & $2.0+0.16 \mathrm{a}$ \\
\hline BMI $(\mathrm{Kg} / \mathrm{m} 2)$ & $36+20.75 \mathrm{a}$ & $42+20.75 \mathrm{a}$ & $35+20.75 \mathrm{a}$ \\
\hline
\end{tabular}

Table 3: Anthropometric Parameters of Malaria infected Pregnant Women on ACT Treatment and Malaria Infected Pregnant Women without ACT Treatment.

Results are expressed as mean and standard deviation. $\mathrm{P} \leq 0.05$ Test values carrying superscript (a) are not significantly different from the control.

Group 1: Malaria infected pregnant women on ACT treatment

Group 2: Malaria infected pregnant women without ACT treatment

Control: Pregnant women without malaria

\section{Discussion}

Results obtained from this study, as displayed in Table 1 show that, the enzymatic antioxidant activities in the plasma of Plamosdium falciparum malarial infected patients on ACT drugs didn't show any significant reduction from the control subjects. However, Table 2 results show significant reduction of all the antioxidants determined in group 2 subjects which is made up of pregnant patients with malaria without ACT treatment. The result here evidently showed that Plasmodium falciparum malarial infection significantly $(\mathrm{p}<0.05)$ reduced enzymatic antioxidant levels, but ACT treatment significantly (p <0.05) improved the enzymatic antioxidant levels. This result is in conformity with the literature. Infection with Plasmodium falciparum has been observed to stimulate the immune system and this has been established to increase the production of free radicals, especially reactive oxygen species, ROS [18]. It therefore implies that, treatment of Plasmodium falciparum malarial infection with ACT boosted the capacity of plasma antioxidants. The mechanism(s) of this ACT - enhanced antioxidant capacity in plasma is complex, but it seems that ACT may elicit the production of antioxidants. Catalase and SOD are two respective important preventive and chain breaking antioxidants [19]. Since ACT drugs target and destroy malarial parasite infected erythrocytes, the immune system produces antioxidants to metabolize the existence of these drugs, thereby causing an increased production and concentration of total enzymatic antioxidants. Treatment of Plasmodium falciparum malarial infection with ACT seems to enhance antioxidants' defense against the parasites' induced increase in ROS reactivity and possible associated damages.

Table 3 results did not show any significant difference in the age, and body mass index (BMI), of all the patients in group 1 and 2 , as compared with the control group. This suggests that malaria infection seems not to be a function of age, weight or height of the patients.

\section{Conclusion}

In conclusion, this study suggests that the use of artemisin combination therapy (ACT) in malaria issues may prevent decrease the enzymatic antioxidants activities of the pregnant women when administered. Hence, having optimum activities of these antioxidants will surely help the pregnant women to stay free of other diseases which can lead to further complications of the pregnancy.

\section{Acknowledgement}

All the Professors in the department of Biochemistry, Ekiti state university, Nigeria and the chief technologists of the university are highly appreciated for their contributions and suggestions that made this research work a success. Also, all the subjects that participated in this research study are appreciated for their consent.

\section{References}

1. Arora DR, Arora B (2008) Medical Parasitology $2^{\text {nd }}$ (Edn.), CBS Publishers and Distributors. PVT LTD. India 3(8): 168-180.

2. Olowe OA, Makanjuola OB, Awa AO, Olowe RA (2015) Malaria in Africa and the Historical Perspective: The Journey so Far. Journal of Biology and Medical Sciences 3: 33-41.

3. World Health Organisation (2010) World Malaria Report 2010, pp: 238.

4. Fievet N, Cot M, Ringwald P, Bickii J, Dubois B, et al. (1997) Immune response to Plasmodium falciparum antigens in Cameroonian primigravidae: evolution 
after delivery and during second pregnancy. Clin Exp Immunol 107(3): 462-467.

5. Raimi OG, Kanu CP (2010) The prevalence of malaria infection in pregnant women living in a suburb of Lagos, Nigeria. African Journal of Biochemistry Research 4(10): 243-245.

6. Greenwood BM (1997) The use of anti-malarial drugs to prevent malaria in the population of malariaendemic areas. Am J Trop Med Hyg 70(1): 1-7.

7. Bulter D (1997) Time to Put Malaria Control on the Global Agenda. Nature 386(6625): 535-541.

8. Molyneux ME, Taylor TE, Wirima JJ, Borgstein A (1989) Clinical Features and Prognostic indicators in pediatric cerebral malaria: A study of 131 comatose Malawian children. QJM 71(265): 369-371.

9. Tiwari BK, Pandey KB, Abidi AB, Rizvi SI (2013) Markers of oxidative stress during diabetes mellitus. J of Biomarker 2013: 1-8.

10. Ogbodo SO, Shu EN, Okeke AC (2006) Vitamin antioxidants may prevent drug induced hemolysis of G6PD-deficient erythrocytes. Pharmacology online 1: 90-99.

11. Toner K, Sojka G, Ellis R (2007) "A Quantitative Enzyme Study; catalase" bucknell. Edu, pp: 1-12.

12. World Health Organization (WHO) (2014) World Malaria Report 2014 fact sheet.

13. Ezenduka CC, Okonta MJ, Esimone CO (2014) Adherence to treatment guidelines for uncomplicated malaria at two public health facilities in Nigeria; Implications for the 'test and treat' policy of malaria case management. J Pharmaceut Policy Pract 7(1): 15 .

14. Obidike IC, Okhale S, Aboh MI, Salawu OA (2013) Isolation, fractionation and evaluation of the antiplasmodial properties of Phyllanthus niruri resident in its chloroform fraction. Asian Pac J Trop Med 6(3): 169-175.

15. Marklund S, Marklund G (1974) Involvement of superoxide anion radical in the autoxidation pf pyrogallol and a convenient assay for superoxide dismutase. Eur J Biochem 47(3): 469-474.

16. Beers RF, Sizer IW (1952) Spectrophotometric method of measuring the breakdown of hydrogen peroxide by catalase. J Biol Chem 195(1): 133-140.

17. Paglia DE, Valentine WN (1967) Studies on the quantitative and qualitative characterization of erythrocyte gluthathione peroxidase. J Lab Clin Med 70(1): 158-169.

18. Kremsner PG, Greve B, Luckner D, Schmid D (2000) Malaria anaemia in African children associated with high oxygen radical production. Lancet 355(9197): $40-41$.

19. Kulkarni AG, Suryakar AN, Sardeshmukh AS, Rathi DB (2003) Studies on biochemical changes with special reference to oxidant and antioxidant in malarial patients. Ind J Clin Biochem 18(2): 136-149. 